\title{
An optimized MLP model to diagnosis the bipolar disorder
}

\author{
Mozhgan Mohammad Ghasemi ${ }^{1 *}$, Mehdi Khalili ${ }^{2}$ \\ ${ }^{1}$ M.S. Student, Department of Computer and Informatics Payame Noor University, Tehran, Iran \\ ${ }^{2}$ Assistant Professor, Department of Computer and Informatics Payame Noor University,Tehran, Iran \\ *Corresponding author E-mail: Mozhgan_7327@yahoo.com
}

Copyright () 2015 Mozhgan Mohammad Ghasemi, Mehdi Khalili. This is an open access article distributed under the Creative Commons Attribution License, which permits unrestricted use, distribution, and reproduction in any medium, provided the original work is properly cited.

\begin{abstract}
The use of artificial neural networks in different areas of engineering science is growing by the day. The significant proportions of research in medical engineering, Therefore in this paper have tried to implemented MLP model with 47 parameters for diagnosis of bipolar disorder. Parameters such as: lack of pleasure, feelings of guilt, worthlessness, lack of success, mental anxiety, somatic anxiety disorder, the disorder of interest, etc. in next part, we done the manipulation structure of MLP model, for this work switching the function in layers. And comparing the error of manipulation structure with previous manipulation. We concluded with using purelin function in layers, the error of diagnosis reduces $4 \%$ and this value is an acceptable value.
\end{abstract}

Keywords: ANN; $M L P ; B D$.

\section{Introduction}

Diagnosis of psychiatric illness always has been one of the most important steps in the treatment of diseases [1]. And with the input of the neural network to diagnose diseases [2], in this paper attempts to identify the optimal diagnosis of bipolar disorder by using the MLP model of neural network. The analysis of scientific investigations shows that very limited works have been reported on diagnosis of depression using neural networks structure, for example: In [3], the authors conducted a resting-state functional connectivity fMRI study of 35 bipolar disorder and 25 schizophrenia patients, to investigate the relationship between bipolar disorder and schizophrenia, using computation of the mean connectivity within and between five neural networks: default mode (DM), fronto-parietal (FP), cingulo-opercular (CO), cerebellar (CER), and salience (SAL). They found that across groups, connectivity was decreased between COCER, to a larger degree in schizophrenia than in bipolar disorder. Also, in schizophrenia, there was also decreased connectivity in CO-SAL, FP-CO, and FP-CER, while bipolar disorder showed decreased CER-SAL connectivity. Disorganization symptoms were predicted by connectivity between CO-CER and CER-SAL. In [4], ten different types of classification algorithms are applied to depression diagnosis and their performance is compared, through a set of experiments on SMRI brain scans. In the experiments, a procedure is developed to measure the performance of these algorithms and an evaluation method is employed to evaluate and compare the performance of the classifiers and concluded that with using SVM model the best classification happens with $85.29 \%$ accuracy.

In this paper, we implemented the proposed method in 2 parts by using MLP model. In first part done with different percentage training and obtain the minimum error and best percentage training, in second part we done the optimized the structure of MLP model. And in the end, comparing the results of both parts.

\section{MLP model}

Multilayer Perceptron network is of the feed forward neural networks, that one of the most widely used models of artificial neural networks in modeling .Multilayer Perceptron network, each neuron in each layer are connected to all neurons in the previous layer [5].In such networks, networks that are fully connected [6]. In "Fig. 1" view of this model is visible. 


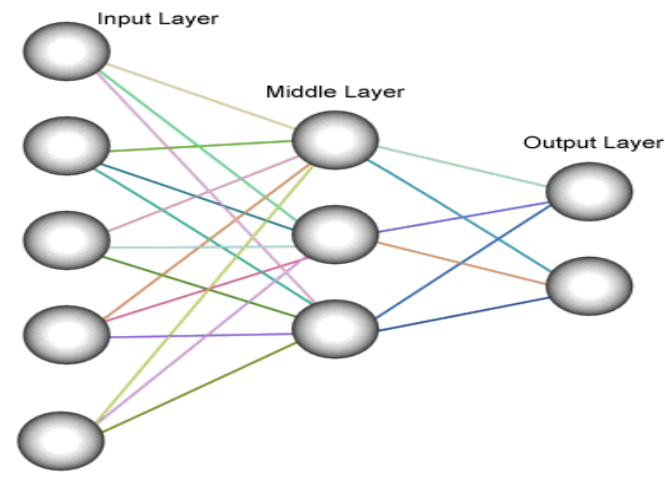

Fig. 1: MLP Model

\section{Depression \& bipolar disorder}

"Depression" is a psychological disorder in which the patient's activities severely reduced and in fact, he was not motivated to do many things. Depressed person's energy and life skills he will fall, and his concentration is greatly reduced. However, sometimes aggressive, sometimes it is frustrating. His guilt is very strong. In addition, the patient will return your goals in life and reduce social activities and production [7]. Bipolar disorder (BD), an unstable emotional condition characterized by cycles of abnormal, persistent high mood (mania) and low mood (depression), which was formerly known as "manic depression" (and in some cases rapid cycling, mixed states, and psychotic symptoms) $[8,9]$. Subtypes include: Bipolar I is distinguished by the presence or history of one or more manic episodes or mixed episodes with or without major depressive episodes [10].

\section{Proposed method}

We describe the proposed method for diagnosis of bipolar disorder. Proposed methods have the 2 part. In two parts using the structure of MLP model. The schema of proposed method shown in "Fig.2", and the details are as follows:

- Data divided in two part, the first part is "train" that use to train network. And second part is "testing" that using in test network.

- MLP model train from part "train" and parameters for different percentage training.

- Output of first part obtained and stored in "OP1" and error of this part stored in "E1".

- The best percentage training that has minimum error, saved in " $T$ ".

- $\quad$ For second part of proposed method, optimized the structure of MLP model, by using “T”. For optimized change the function in three layers (input, middle, and output).

- Output of second part saved in "OP2" and error of this part stored in "E2".

- For choose the best output, compare the error of both part. That shown in "Eq.1":

$\{O P 2$

$\{O P 1$

$$
\begin{aligned}
& E 1 \geq E 2 \\
& \text { O. } W
\end{aligned}
$$

\section{Experiment}

The number of parameters used in this paper has 47 parameters, these include: lack of pleasure, feelings of guilt, worthlessness, lack of success, mental anxiety, somatic anxiety disorder, the disorder of interest, impaired appetite, suicidal thoughts, insomnia, excessive happiness, distraction, Jabber talking too fast, increasing social activity, jump thoughts, poor judgment, educational activity, dissatisfaction, lavish use of stimulants, slow motion, decreased libido, increase energy, lack of concentration, sex risk, investment risk, increase the time for studying [11], [12]. The experiments of both parts were implemented using MATLAB 8.1. The two parts are described below. 


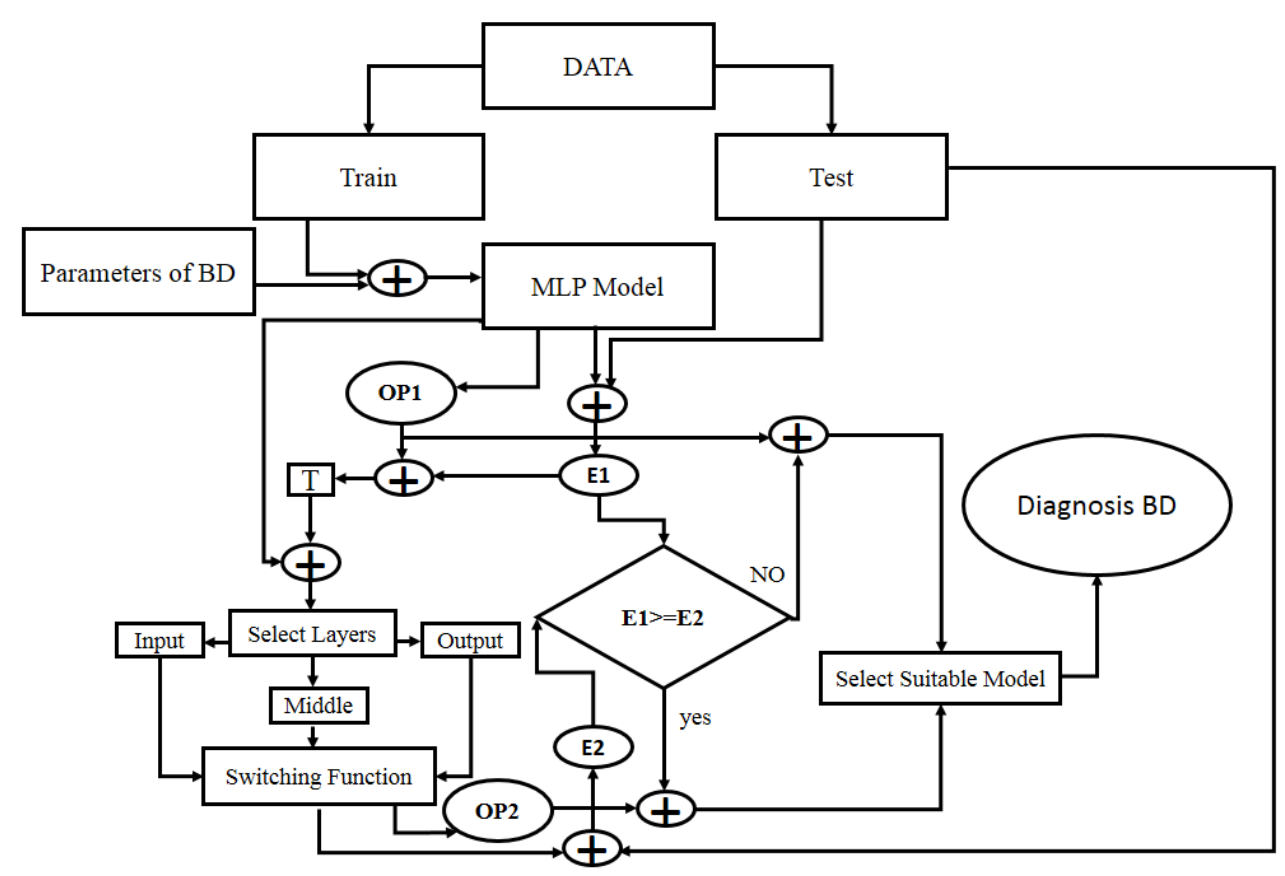

Fig. 2: Proposed Method

\section{The first part of the experiments}

First part implemented with using 10, 5, 4 neurons respectively in input, middle and output layer. This part simulation with the different percentage training. Results of this part shown in "Table 1". As can be seen when using $70 \%$ of data for training network have the minimum error, this error is 0.16 and value of $\mathrm{T}$ is $70 \%$, also $\mathrm{E} 1=0.16$. By using $60 \%$ of data for training network have the maximum error $(0.22)$ ?

The regression graph as can be seen in "Fig.3". Regression graph shown, when using all data (train, test and validation) for testing network, the output of network how is consistent with target of network. I t is obvious; whatever line of figure is closer to 1 , that is better, because the regression graph has the value between 0 to 1 . That the value of regression in this part is 0.90 .

Table 1: Error of First Part

\begin{tabular}{ll}
\hline Network error & Percentage training \\
\hline 0.19 & 40 \\
0.19 & 50 \\
0.22 & 60 \\
0.16 & 70 \\
0.17 & 80 \\
0.19 & 90 \\
\hline
\end{tabular}

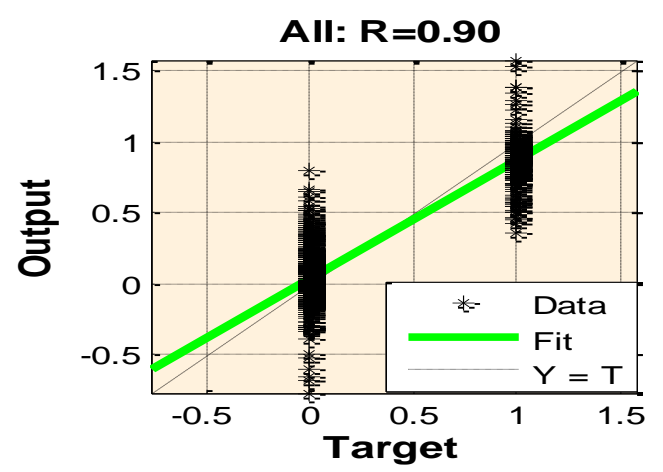

Fig. 3: Regression of First Part. 


\section{The second part of the experiments}

In this part for optimized, we manipulation the structure of MLP model. We change the function that uses in layers. Functions that the used in layers, included: Tansig, Purelin or Logsig. The percentage training select of previous part that stored in T. "Table.2" showed the changing function and results. As can be seen when using Purelin function in three layer we have the minimum error, this error is 0.04 (E2=0.04). And when using the purelin in input layer, Logsig in middle layer and Tansig in output layer.

The regression graph as can be seen in "Fig.4". Regression graph shown, when using all data (train, test and validation) for testing network, the output of network how is consistent with target of network. I t is obvious; whatever line of figure is closer to 1 , that is better, because the regression graph has the value between 0 to 1 . That the value of regression in this part is 0.96 .

Table 2: Error of Second Part

\begin{tabular}{ll}
\hline Error network & Network structure \\
\hline 0.22 & Tansig Tansig Tansig \\
0.04 & Purelin Purelin Purelin \\
0.20 & Tansig Logsig Purelin \\
0.17 & Tansig Purelin Logsig \\
0.36 & Purelin Logsig Tansig \\
0.34 & Purelin Tansig Logsig \\
0.18 & Logsig Purelin Tansig \\
0.16 & Logsig Logsig Logsig \\
\hline
\end{tabular}

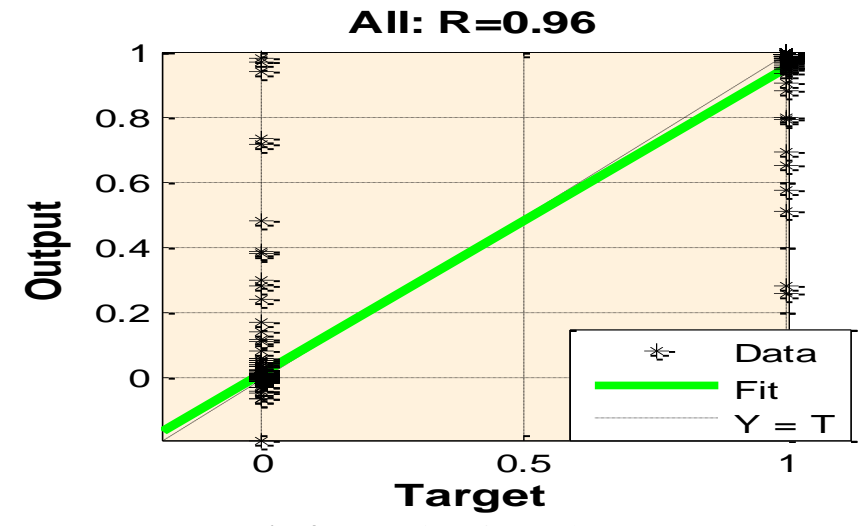

Fig. 4: Regression of Second Part

\section{Choose the best part}

In this section, implemented the end part of proposed method for choosing the model with minimum error for diagnosis bipolar disorder. This comparing shown in "Fig.5". As can be seen by using the second parts have the minimum error (0.04) than first part, and using the "OP2" for diagnosis.

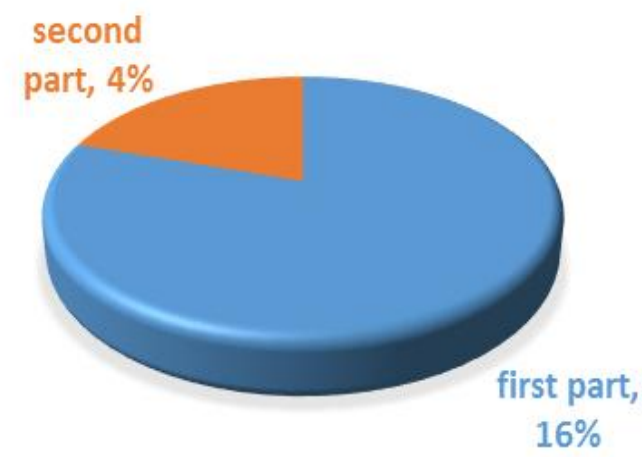

Fig. 5: Compare the Parts 


\section{Conclusion}

In this paper, one proposed method definition for diagnosis of bipolar disorder. This method including the 2 part. In first part pay to implemented MLP model by using the different percentage training for get the best percentage training for using second part. In second part done the manipulation MLP model for reduce the error of this model. Then, the error of both parts comparing and select the suitable output. That error of second part equal to $4 \%$. This error is a very good for primary diagnosis of bipolar disorder.

\section{References}

[1] Bauer.M, Pfennig.A, Severus.E, etc, "World Federation of Societies of Biological Psychiatry (WFSBP) guidelines for biological treatment of unipolar depressive disorders", vol. 14, The World Journal of Biological Psychiatry, 2013.

[2] Fukushima, K., "Neocognitron: A self-organizing neural network model for a mechanism of pattern recognition unaffected by shift in position". Biological Cybernetics, vol. 36 (4), 2014, pp. 93 http://dx.doi.org/10.1016/j.jad.2013.01.051.

[3] Mamah, D; Barch, DM.”Resting state functional connectivity of five neural networks in bipolar disorder and schizophrenia”. J Affect Disord; DOI. 10.1016/j.jad.2013.01.051. 2013. Pp.214-223.

[4] Kipli and Y.Xiang, "An Empirical Comparison of Classification Algorithms for Diagnosis of Depression from Brain SMRI Scans", Advanced Computer Science Applications and Technologies (ACSAT), pp. 333-336, 2013.

[5] phil.picton, "introduction to neural network", Macmillan publishers limited, 1994

[6] Filippo.Amato, Alberto.López, etc, “Artificial Neural Networks in Medical Diagnosis”, Vol. 11, Journal of Applied Biomedicine, 2013.

[7] G. O. Young, "Synthetic structure of industrial plastics (Book style with paper title and editor)," in Plastics, 2nd ed. vol. 3, J. Peters, Ed. New York: McGraw-Hill, 1964, pp. 15-64.

[8] Zimmerman. M. "Would broadening the diagnostic criteria for bipolar disorder do more harm than good? Implications from longitudinal studies of subthreshold conditions", Vol. 73(4), J Clin Psychiatry, 2012, pp.437-443. http://dx.doi.org/10.4088/JCP.11com07288.

[9] Anderson, IM; Haddad, PM; Scott, "Bipolar disorder." BMJ (Clinical research ed.), 2012, pp. 345.

[10] Parry, P.I.; Levin, E.C., "Pediatric Bipolar Disorder in an Era of "Mindless Psychiatry"". Journal of Trauma \& Dissociation, VOL. 13 (1), 2013, PP. 51-68.

[11] Mana S, Martinot MLP, Martinot JL," Brain Imaging Findings in Children and Adolescents with Mental Disorders", a Cross-sectional Review J Eurpsy, VOL. 25, 2013, PP. 345-354.

[12] Pfennig.A, Bschor.T, Baghai.T, etc. "S3 guidelines ondiagnostics and therapy of bipolar disorders: developmentprocess andessential recommendations", Vol.83 (5), Nervenarzt, 2012, pp.568-586. 